\title{
Absence of helical surface states in bulk semimetals with broken inversion symmetry
}

\author{
Carmine Ortix ${ }^{1}$ Jörn W.F. Venderbos,${ }^{1}$ Roland Hayn,${ }^{2}$ and Jeroen van den Brink ${ }^{1}$ \\ ${ }^{1}$ Institute for Theoretical Solid State Physics, IFW Dresden, D01171 Dresden, Germany \\ ${ }^{2}$ Aix-Marseille Univ., CNRS, IM2NP-UMR 7334, 13397 Marseille Cedex 20, France
}

(Dated: August 28, 2021)

\begin{abstract}
Whereas the concept of topological band-structures was developed originally for insulators with a bulk bandgap, it has become increasingly clear that the prime consequences of a non-trivial topology - spin-momentum locking of surface states - can also be encountered in gapless systems. Concentrating on the paradigmatic example of mercury chalcogenides $\mathrm{HgX}(\mathrm{X}=\mathrm{Te}$, Se, $\mathrm{S})$, we show that the existence of helical semimetals, i.e. semimetals with topological surface states, critically depends on the presence of crystal inversion symmetry. An infinitesimally small broken inversion symmetry (BIS) renders the helical semimetallic state unstable. The BIS is also very important in the fully gapped regime, renormalizing the surface Dirac cones in an anisotropic manner. As a consequence the handedness of the Dirac cones can be flipped by a biaxial stress field.
\end{abstract}

PACS numbers: 73.20.At,71.55.Gs, 72.80.Sk

Introduction - The discovery of two- and threedimensional (3D) topological insulators (TIs) 1-15 has brought to light a new state of quantum matter. This has had a tremendous impact in the field of fundamental condensed matter physics as well as for potential applications in spintronics and quantum computation [16]. The TIs are insulating in the bulk but have topologically protected surface states [2, 4, 14, and the topology dictates that the metallic surface states are spinmomentum locked: surface electrons with opposite spin counter-propagate at the sample boundaries [3, 4, 7, 9,

Materials with a TI band structure such as $\mathrm{Sb}$ [13, $\mathrm{Bi}_{2} \mathrm{Se}_{3}$ [17] and $\mathrm{Bi}_{14} \mathrm{Rh}_{3} \mathrm{I}_{9}$ [15] often show the presence of a finite bulk carrier density. In such materials, the bulk Fermi surface does not simply swallow up the topological surface states. They survive and coexist with a bulk Fermi surface [13, leading to the notion of a helical metal. The coexistence of a bulk Fermi surface and topological surface states can be understood as a doped TI being made out of a bulk TI with a non-topological metallic band inside the gap. The hybridization between the topological surface states and the additional metallic band pushes the topological surface states away from overlapping with the bulk states in energy and momentum. This preserves them in a slightly modified form at those points in the Brillouin zone (BZ) where the surface and the bulk bands do not cross [18. Also in bulk semimetals with a topological non-trivial band ordering surface Dirac-like states are expected to coexist with metallic states [19, 20], suggesting the analogous presence of a helical semimetallic state.

Using the paradigmatic example of the series of cubic mercury chalcogenides $\mathrm{HgX}(\mathrm{X}=\mathrm{Te}, \mathrm{Se}, \mathrm{S})$, we show however that the existence of a helical semimetallic state critically relies on the presence of crystal inversion symmetry even in the absence of disorder. An infinitesimally small broken inversion symmetry (BIS) is detrimental for the topological surface states of a helical semimetal, in-
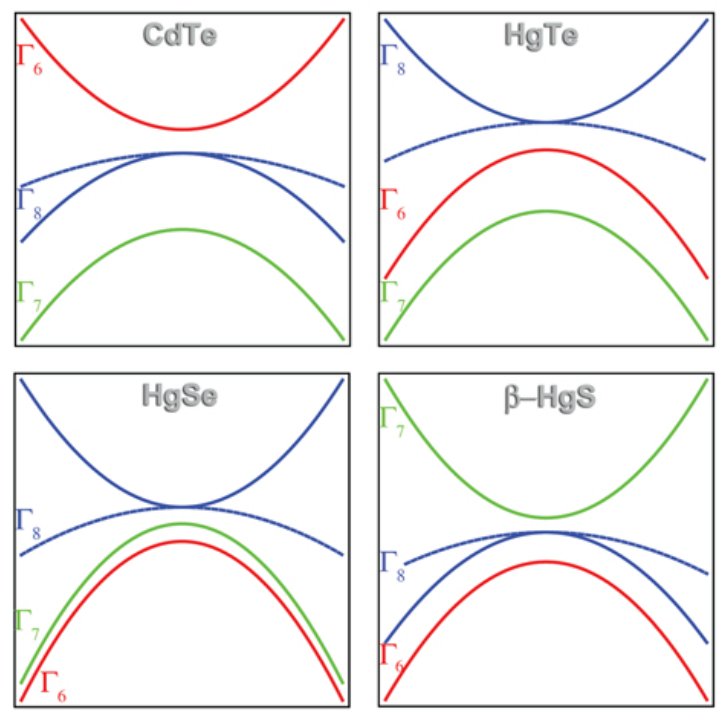

FIG. 1: Schematic band-structure close to the Brillouin zone centre of the cubic mercury chalcogenides inverted semiconductors $\mathrm{HgX}$ as compared to the topologically trivial CdTe semiconductor.

dependent of any overlap in energy and momentum of bulk and topological surface states. We show furthermore that in the fully gapped TI regime, a BIS does not endanger the existence of topological surface states. In this case the BIS rather renormalizes the Fermi velocity of the surface Dirac fermions in an anisotropic manner, similarly to the effect envisioned in anisotropic graphene superlattices [21, 22. This in principle allows an externally applied biaxial stress field to flip the surface state chirality in a material with BIS.

$\mathrm{HgX}$ compounds - Pristine $\mathrm{HgTe}$ is a semimetal which is charge neutral when the Fermi energy is at the touching point between the light-hole ( $\mathrm{LH})$ and the heavy-hole (HH) $\Gamma_{8}$ bands at the BZ center 20, 23. The topologi- 
cal nature of the electronic states in this material cannot be inferred from these $p_{3 / 2}$ atomic levels but rather follows from the inverted band ordering at the zone center of the $\mathrm{LH} \Gamma_{8}$ band which is particle-like and the $\Gamma_{6} s$ band which is hole-like. In normal semiconductors, such as CdTe [see Fig.1], $\Gamma_{6}$ forms the conduction band and $\Gamma_{8}$ is one of the valence bands. The consequence of this band inversion can be understood from the simple criterion derived by $\mathrm{Fu}$ and Kane [6] to distinguish normal and non-trivial topological classes. This criterion, which relies on the presence of inversion symmetry, establishes a material to be in a topologically non-trivial class if two bands of opposite parity have level-crossed with respect to the normal band ordering. The zinc-blende crystal structures of HgTe lacks inversion symmetry, but it is normally considered that the BIS acts as a small perturbation and, by invoking the principle of adiabatic continuity, does not hinder the topological nature of the level crossing. As the $\mathrm{HH}$ bands do not participate in the topological level crossing, it can be assumed that they act as inserted "parasitic" bulk bands closing the full band gap and preventing the system to be a strong $3 \mathrm{D}$ TI. It is expected [19, 20] that the existence of topological surface states resulting from the $\mathrm{LH}-\Gamma_{6}$ TI bulk is not undermined by the presence of the $\mathrm{HH}$ bulk bands suggesting $\mathrm{HgTe}$ to be a helical semimetal.

Similar arguments apply to HgSe which has the same band ordering as $\mathrm{HgTe}$ but with the difference that the spin-orbit (SO) split-off $\Gamma_{7}$ bands are above the $\Gamma_{6}$ bands 24] - the SO splitting $\Delta_{0}=E\left(\Gamma_{8}\right)-E\left(\Gamma_{7}\right)$ is smaller than the gap $-E_{0}=E\left(\Gamma_{8}\right)-E\left(\Gamma_{6}\right)$ [c.f. Fig. 1]. In this case the SO split-off bands, the LH and the $\Gamma_{6}$ bands realise a bulk TI with the $\mathrm{HH}$ bands playing as in $\mathrm{HgTe}$ the role of parasitic bands which close the full band gap. Yet another material of the same family - metacinnabar - was proposed to be in a topologically non-trivial class: a recent fully-relativistic electronic structure calculation 25] finds the required band-ordering, although in this particular case the $\Gamma_{7}$ bands and $\Gamma_{6}$ have switched places with respect to the normal ordering [c.f. Fig. 1] and thus $\Delta_{0}<0$. This reversed order originates from a small but significant contribution of $\mathrm{Hg} 5 d$ orbitals whose spin-orbit (SO) coupling dominates over the sulfur $3 p$ states and reverses its sign 26 by an amount sufficient enough to create a small gap thus rendering $\beta-\mathrm{HgS}$ a stoichiometric strong 3D TI .

Inversion invariant effective Hamiltonian - For an analysis of the topological surface states one can rely on effective low-energy theories that are based upon a $\mathbf{k} \cdot \mathbf{p}$ expansion of the lowest energy bands around the $\Gamma$ point. In the simplest case, as for instance $\mathrm{Bi}_{2} \mathrm{Se}_{3}$, the $\mathbf{k} \cdot \mathbf{p}$ theory yields a Dirac-like Hamiltonian [9, 27] with the Dirac-mass representing the energy gap and a change of its sign corresponding to a nontrivial level crossing. The same approach applies to the mercury chalcogenides and has correctly predicted the Quantum Spin Hall effect in (a)

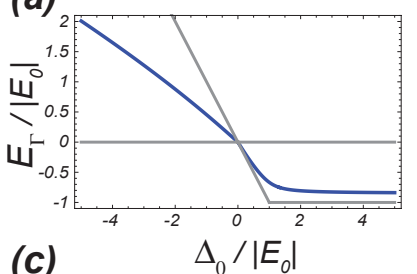

(b)

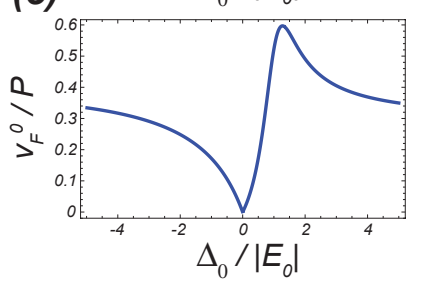

(d)
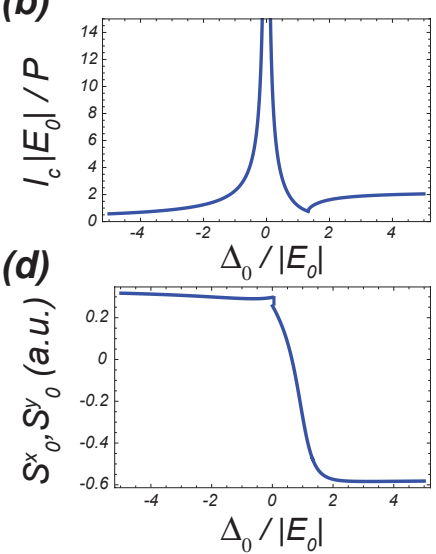

FIG. 2: (a) Behavior of the energy of the surface Dirac point measured in units of the gap $\left|E_{0}\right|$ as a function of the ratio among the spin-orbit splitting $\Delta_{0}$ and $\left|E_{0}\right|$ in the absence of broken inversion symmetry. The gray lines correspond to the conduction and valence bulk band-edges by artificially removing the parasitic $\mathrm{HH}$ bands. (b) Behavior of the decay length for the surface states at the centre of the surface Brillouin zone as a function of $\Delta_{0} /\left|E_{0}\right|$. (c) Same for the Fermi velocity of the surface Dirac cones $v_{F}^{0}$ measured in units of the band structure parameter $P$. (d) Behavior of the spin constants $S_{0}^{x, y}$ as a function of the spin-splitting energy $\Delta_{0}$.

$\mathrm{HgTe} / \mathrm{CdTe}$ quantum wells 3 . The generic form of a low-energy $\mathbf{k} \cdot \mathbf{p}$ expansion at the BZ center $\Gamma$ for semiconductors with a zinc-blende crystal structure is given by the Kane model Hamiltonian 28. Previous work has focused predominantly on the bands responsible for the level crossing, the $\Gamma_{6}$ and $\Gamma_{8}$ bands [20, 23]. While such an analysis is capable of correctly describing the topological characteristics of $\mathrm{HgTe}$ and its consequences for surface excitations, here we consider the full eight-band Kane model Hamiltonian which takes into account the $\Gamma_{6}, \Gamma_{7}$ and $\Gamma_{8}$ bands and correctly describes the band ordering near the Brillouin zone centre of the series of mercury chalcogenides $\mathrm{HgX}$ once the spin-orbit splitting energy $\Delta_{0}$ is varied. This allows us to smoothly connect from the intrinsic, fully gapped, TI regime realised in $\beta$ $\mathrm{HgS}$ to the putative helical semimetal regime for $\Delta_{0}>0$ and analyse the fate of the resulting topological surface states. Even more $\Delta_{0}$ not only provides a convenient tuning parameter, its variation represents the physical effect of biaxial strain fields. Simultaneous application of two stress fields directed along the [100] ([010]) and [001] directions will under specific conditions [see the Supplemental Material] preserve the degeneracy at the $\Gamma$ point among the $\mathrm{LH}$ and $\mathrm{HH}$ bands, but renormalize the SO energy $\Delta_{0}$.

To establish the helical semimetal state in the cubic mercury chalcogenides when inversion symmetry is preserved, we explicitly calculate the [001] surface states of the eight-band Kane model Hamiltonian by neglecting BIS effects on the half-space $z>0$ [27] with open bound- 
ary conditions. For illustration of the physics we take for simplicity the $\mathrm{HgTe}$ band structure parameters at $T=0 \mathrm{~K} 29$. At the $\Gamma$ point of the surface BZ, the Kane model Hamiltonian predicts the $\mathrm{HH}$ bands to be completely decoupled from the other bands. This decoupling guarantees the absence of any mixing among the parasitic $\mathrm{HH}$ bands and the topological surface states resulting from the TI bulk. The corresponding part of the Hamiltonian is block diagonal with the two blocks for the chalcogen $p$-type (mercury $s$-type) states of total angular momentum $J_{z}=1 / 2$ and $J_{z}=-1 / 2$ respectively. The eigenstates have therefore the form $\Psi^{\uparrow}(z)=\left(\psi_{0}^{\uparrow}, \mathbf{0}\right)^{T}$ and $\Psi^{\downarrow}(z)=\left(\mathbf{0}, \psi_{0}^{\downarrow}\right)^{T}$ where $\psi_{0}^{\uparrow, \downarrow}$ is a three-dimensional spinor and $\mathbf{0}$ is a five component zero vector. For the surface states, the wavefunction $\psi_{0}^{\uparrow, \downarrow}(z)$ is localized at the [001] surface in which case $\Psi^{\uparrow, \downarrow}$ play the role of a spin one-half surface Kramer's doublet [see the Supplemental Material].

Fig 2(a) shows the energy of the surface Kramer's doublet as a function of the ratio among the SO splitting energy $\Delta_{0}$ and the $\Gamma_{6}-\Gamma_{8}$ gap $-E_{0}$. In the intrinsic, fully gapped, TI regime, $\Delta_{0}<0$, the surface state's energy resides in the direct bulk insulating gap at the $\Gamma$ point. In the $\Delta_{0}<0$ regime, instead, the surface Kramer's doublet energy lies below the zero energy $\mathrm{HH}$ band-edge but resides in the band-gap of the TI bulk realised by the $\Gamma_{6,7}$-LH bands. Fig,2(b) shows the behavior of the decay length of the surface states. We find that precisely at $\Delta_{0} \equiv 0$ - where the gap of the bulk TI closes - the decay length diverges and thus the condition for the existence of the surface states is violated. For finite values of the spin-orbit splitting, instead, the existence condition for the surface states is fulfilled which is guaranteed by the fact that a renormalizable surface state solution exists in the half-infinite space $z>0$. By projecting the bulk Hamiltonian onto the subspace of these two surface states [9], we obtain an effective surface Hamiltonian to the leading order of $k_{x, y}$

$$
\mathcal{H}_{\text {surf }}\left(k_{x}, k_{y}\right)=E_{\Gamma} \mathcal{I}+v_{F}^{0}\left(\sigma_{x} k_{y}-\sigma_{y} k_{x}\right),
$$

with the Fermi velocity $v_{F}^{0}$ whose behavior as a function of the spin-orbit splitting is shown in Fig[2(c). That the $\sigma$ matrices in the effective surface model Hamiltonian are proportional to the real spin can be shown by projecting the total angular momentum operators $J_{x, y, z}$ onto the surface state subspace. Independent of the spinorbit splitting energy, we do find that $\left\langle\Psi\left|J_{x, y, z}\right| \Psi\right\rangle \equiv$ $S_{0}^{x, y, z} \sigma_{x, y, z}$ where $S_{0}^{z} \equiv 1 / 2$ whereas $S_{0}^{x} \equiv S_{0}^{y}$ with a finite value whose behavior as a function of $\Delta_{0}$ is shown in Fig 2(d). As a result, the surface states show a linear dispersion with helical spin-textures left-handed for the surface conduction band and right-handed for the surface valence band proving the spin-momentum locking of the surface state solutions.

Broken inversion symmetry - Having established that in presence of inversion symmetry, the series of cubic mer-
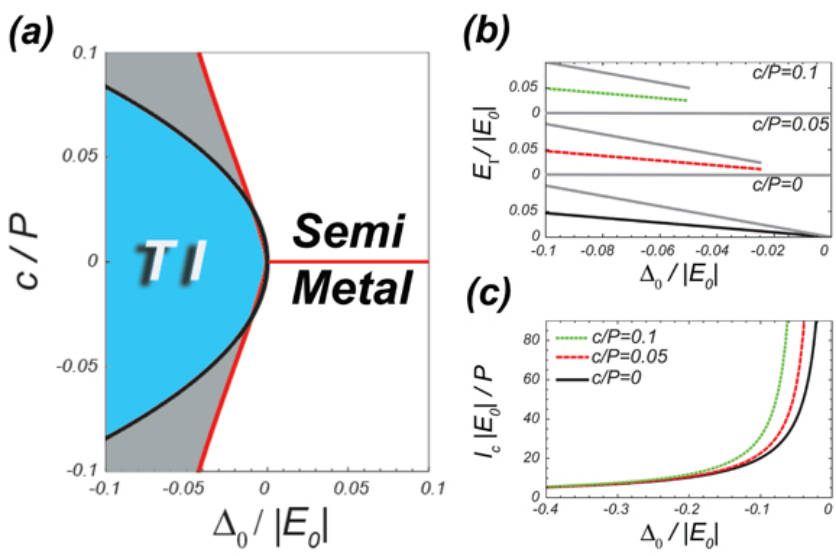

FIG. 3: (a) Phase diagram for the existence of renormalizable surface state solutions in the [001] surface of $\mathrm{HgX}$ mercury chalcogenide compounds as obtained from the eightband Kane model Hamiltonian. The gray area corresponds to regions with topological surface states at the BZ centre where the opening of the indirect bulk band gap cannot be correctly captured by the Kane model . (b) Behavior of the energy of the surface Dirac point measured in units of the gap $\left|E_{0}\right|$ as a function of the ratio among the spin-orbit splitting $\Delta_{0}$ and $\left|E_{0}\right|$ for different strengths of the linear in $\mathbf{k}$ BIS terms. The Dirac point energy always lies in the gap at the $\Gamma$ point among the $\Gamma_{8}$ and the SO split-off bands $\Gamma_{7}$ whose band-edges are represent by the gray lines. (c) Same for the decay length of the surface states.

cury chalcogenides will either be in the strong 3D TI or in the helical semimetal state, we now take into account the intrinsic BIS of the zinc-blende crystal structure. From a k.p perspective, the BIS allows for additional terms in the bulk Hamiltonian once the point group symmetry is reduced to $D_{2 d}$ [23, 28]. In the valence band block $\mathcal{H}^{8,8}$ of the Kane model Hamiltonian [see the Supplemental Material] the BIS indeed yields an additional term [30] $\mathcal{H}_{B I S}^{8,8}=c\left[\left\{J_{x}, J_{y}^{2}-J_{z}^{2}\right\} k_{x}+\right.$ c.p. $] / \sqrt{3}$ and a similar term in the $\mathcal{H}^{8,7}$ block $\mathcal{H}_{B I S}^{8,7}=-i \sqrt{3} c\left[T_{y z}^{\dagger} k_{x}+\right.$ c.p. $]$. The presence of this linear in $\mathbf{k}$ additional terms stems from bilinear terms consisting of k.p and SO interaction with the uppermost $d$ core levels 31. As a result, the parameter $c$ is an elementary parameter of the Kane model that unlike the higher order spin splitting terms induced by BIS cannot be expressed in terms of the extended Kane model [28]. Because of the smallness of the elementary parameter $c \simeq 80 \mathrm{meV} \AA 31$ as compared to the linear parameter coupling $P \simeq 8 \mathrm{eV} \AA$ for $\mathrm{HgTe}$, the conventional wisdom [29] is that the BIS effect is very small in mercury chalcogenides and can be therefore safely neglected.

We do find, however, that the BIS has drastic consequences on both the existence conditions and the dispersion of the surface states. Independent of the actual $c$ value, indeed, the BIS-induced linear in $\mathbf{k}$ terms couple the $\mathrm{HH}$ with the TI bulk at the $\Gamma$ point of the surface BZ. 
(a)

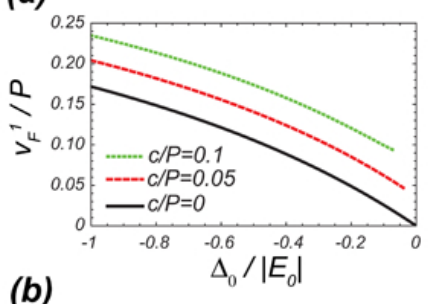

(b)

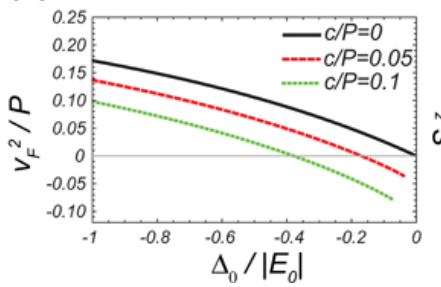

(d)

(c)
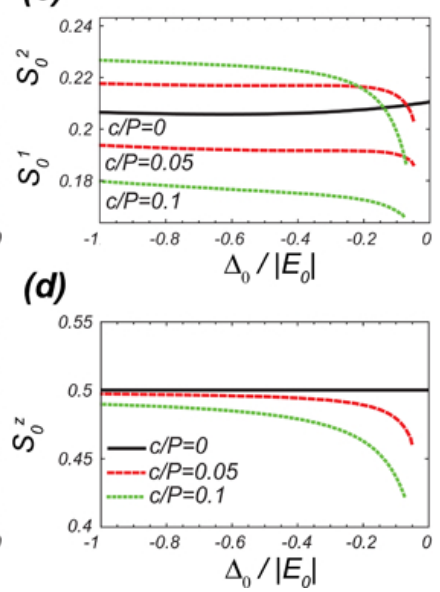

FIG. 4: (a),(b) Behavior of the two non-equivalent Fermi velocities of the surface Dirac cones as a function of the spinorbit splitting $\Delta_{0}$ for different values of the elementary parameter $c$ of the eight-band Kane model Hamiltonian. (c),(d) Same for the behavior of the spin constants $S_{0}^{1,2, z}$.

As a result, the BIS leads to an effective hybridization among the topological surface states and the parasitic $\mathrm{HH}$ bands. One would then expect that whenever the topological surface states overlap in momentum and energy with the parasitic HH bands they should be pushed away. And indeed we find that for positive values of the spin-orbit splitting $\Delta_{0}$, in which case the energy of the surface Kramer doublet lies below the zero-energy $\mathrm{HH}$ band-edge, localized surface state wavefunctions $\Psi^{\uparrow, \downarrow}$ at the $\mathrm{BZ}$ centre do not exist. In the $\Delta_{0}<0$ regime instead, the BIS-induced hybridization should not be effective at the $\Gamma$ point from which one would expect that the existence of the surface Kramer's doublet should not be hampered independent of the actual values of the $c$ parameter and the spin-orbit splitting. On the contrary we find that the existence of topological surface states is intrinsically related to the strength of the linear in $\mathbf{k}$ BIS terms and leads to the phase diagram shown in Fig]3(a). Remarkably for small values of the BIS parameter $c$, renormalizable surface states appear only whenever the spin-orbit splitting is negative by an amount sufficient to create a full indirect band gap. Thus, even in the absence of an overlap in momentum and energy with the parasitic $\mathrm{HH}$ bands, the topological surface states are prevented in the absence of a full bulk band-gap proving that the helical semimetal state is completely suppressed by the BIS.

Fig 3(b) shows the behavior of the surface Kramer's doublet energy at the surface Brillouin zone centre for different values of the BIS elementary parameter $c$ when the intrinsic, fully gapped, TI regime is reached. It always lyes in the bulk gap at the zone centre among the $\Gamma_{8}$ and the SO split-off $\Gamma_{7}$ bands. We also show [c.f. Fig 3 (c)] the behaviour of the penetration depth of the surface states which, increasing the value of the spin- orbit splitting $\Delta_{0}$, increases and eventually diverges at the "topological phase transition" of the phase diagram in Fig. 3(a). By projecting again the bulk Hamiltonian onto the subspace of the surface BZ surface states, we obtain that in the presence of BIS terms the effective surface Hamiltonian to the leading order of $k_{x, y}$ reads

$$
\mathcal{H}_{\text {surf }}\left(k_{x}, k_{y}\right)=E_{\Gamma} \mathcal{I}+v_{F}^{1} k_{1} \sigma_{1}-v_{F}^{2} k_{2} \sigma_{2},
$$

where $k_{1,2}=\left(k_{x} \pm k_{y}\right) / \sqrt{2}$ and $\sigma_{1,2}$ are the corresponding rotated Pauli matrices $\sigma_{1,2}=\left(\sigma_{x} \mp \sigma_{y}\right) / \sqrt{2}$. As a result the surface Dirac cones are anisotropic $\left(v_{F}^{1} \neq v_{F}^{2}\right)$ along the diagonal directions of the surface $\mathrm{BZ}$ as can be shown by a two-dimensional k.p analysis [see the Supplemental Material] and in perfect agreement with the density functional electronic structure calculations in $\beta$ $\mathrm{HgS}$ [25]. In addition, the spin-momentum locking of the surface states is guaranteed by the fact that by projecting the $\pi / 4$ rotated total angular momentum operators $J_{1,2, z}$ onto the subspace of the surface states at the BZ centre we find $\left\langle\Psi\left|J_{1,2, z}\right| \Psi\right\rangle \equiv S_{0}^{1,2, z} \sigma_{1,2, z}$ with $S_{0}^{1,2, z}$ some constants the behaviour of which, as function of the spinorbit splitting $\Delta_{0}$ is shown in Fig 4(c),(d). Fig 4(a),(b) show the behaviour of the two inequivalent Fermi velocities for different values of the BIS parameter $c$. It is evident that for $\Delta_{0} \ll E_{0}$, the surface Dirac cone is strongly anisotropic with a large dispersion along the diagonal $k_{1}$ and a nearly flat band along the perpendicular direction. By varying the strength of the spin-orbit splitting energy, we find a critical value of the spin-orbit splitting $\Delta_{0}^{c}$ where the degree of anisotropy $v_{F}^{1} / v_{F}^{2}$ diverges and the surface states will be completely one-dimensional. Even more, the fact that only one of the two non-equivalent Fermi velocities changes sign, implies a change in the handedness of the surface Dirac cone - left-handed for $\Delta_{0}<\Delta_{0}^{c}$ and right-handed for $\Delta_{0}>\Delta_{0}^{c}$ in the surface conduction band. Therefore, a suitable application of anisotropic biaxial stresses can induce a flip of chirality which would immediately manifest itself as a sign change of the quantized Hall conductance in the presence of a time-reversal symmetry breaking perturbation at the surface.

Conclusions - Coexistence of bulk metallic states with topological surface states can be encountered in a large class of materials. Inverted zero-gap semiconductors fall into this class and provide a prominent example of an helical semimetal. We have shown here that while such a topological state of matter can be established in crystals with inversion symmetry, a breaking of the bulk inversion symmetry initiates a bulk-surface state struggle in which the topological surface states, and thereby the helical semimetallic state as a whole, perish. In the intrinsic, fully gapped, TI regime, the broken inversion symmetry strongly renormalizes the anisotropy of the group velocity of the surface Dirac fermions similarly to graphene superlattices [21, 22]. This might be a relevant feature for spin conduction experiments where a large anisotropy 
of the surface Dirac cone has been predicted to lead to very large spin lifetimes 32 .

The authors thank M. Richter and F. Virot for very fruitful discussions.

[1] C. L. Kane and E. J. Mele, Phys. Rev. Lett. 95, 226801 (2005).

[2] C. L. Kane and E. J. Mele, Phys. Rev. Lett. 95, 146802 (2005).

[3] B. A. Bernevig, T. L. Hughes, and S.-C. Zhang, Science 314, 1757 (2006).

[4] C. Wu, B. A. Bernevig, and S.-C. Zhang, Phys. Rev. Lett. 96, 106401 (2006).

[5] M. König, S. Wiedmann, C. Br?ne, A. Roth, H. Buhmann, L. W. Molenkamp, X.-L. Qi, and S.-C. Zhang, Science 318, 766 (2007).

[6] L. Fu and C. L. Kane, Phys. Rev. B 76, 045302 (2007).

[7] L. Fu, C. L. Kane, and E. J. Mele, Phys. Rev. Lett. 98, 106803 (2007).

[8] J. E. Moore and L. Balents, Phys. Rev. B 75, 121306 (2007).

[9] H. Zhang, C.-X. Liu, X.-L. Qi, X. Dai, Z. Fang, and S.-C. Zhang, Nat Phys 5, 438 (2009).

[10] D. Hsieh, D. Qian, L. Wray, Y. Xia, Y. S. Hor, R. J. Cava, and M. Z. Hasan, Nature 452, 970 (2008).

[11] Y. Xia, D. Qian, D. Hsieh, L. Wray, A. Pal, H. Lin, A. Bansil, D. Grauer, Y. S. Hor, R. J. Cava, and M. Z. Hasan, Nat Phys 5, 398 (2009).

[12] Y. L. Chen, J. G. Analytis, J.-H. Chu, Z. K. Liu, S.-K. Mo, X. L. Qi, H. J. Zhang, D. H. Lu, X. Dai, Z. Fang, S. C. Zhang, I. R. Fisher, Z. Hussain, and Z.-X. Shen, Science 325, 178 (2009).

[13] D. Hsieh, Y. Xia, L. Wray, D. Qian, A. Pal, J. H. Dil, J. Osterwalder, F. Meier, G. Bihlmayer, C. L. Kane, Y. S. Hor, R. J. Cava, and M. Z. Hasan, Science 323, 919 (2009).

[14] M. Z. Hasan and C. L. Kane, Rev. Mod. Phys. 82, 3045 (2010).

[15] B. Rasche, A. Isaeva, M. Ruck, S. Borisenko, V. Zabolotnyy, B. Büchner, K. Koepernik, C. Ortix, M. Richter, and J. van den Brink, Nat Mater 12, 422 (2013).

[16] A. R. Akhmerov, J. Nilsson, and C. W. J. Beenakker, Phys. Rev. Lett. 102, 216404 (2009).

[17] K. Eto, Z. Ren, A. A. Taskin, K. Segawa, and Y. Ando, Phys. Rev. B 81, 195309 (2010).

[18] D. L. Bergman and G. Refael, Phys. Rev. B 82, 195417 (2010).

[19] C. Brüne, C. X. Liu, E. G. Novik, E. M. Hankiewicz, H. Buhmann, Y. L. Chen, X. L. Qi, Z. X. Shen, S. C. Zhang, and L. W. Molenkamp, Phys. Rev. Lett. 106, 126803 (2011).

[20] R.-L. Chu, W.-Y. Shan, J. Lu, and S.-Q. Shen, Phys. Rev. B 83, 075110 (2011).

[21] C.-H. Park, L. Yang, Y.-W. Son, M. L. Cohen, and S. G. Louie, Nat. Phys 4, 213 (2008).

[22] C.-H. Park, Y.-W. Son, L. Yang, M. L. Cohen, and S. G. Louie, Nano Letters 8, 2920 (2008).

[23] X. Dai, T. L. Hughes, X.-L. Qi, Z. Fang, and S.-C. Zhang, Phys. Rev. B 77, 125319 (2008).

[24] A. Svane, N. E. Christensen, M. Cardona, A. N. Chantis,
M. van Schilfgaarde, and T. Kotani, Phys. Rev. B 84, 205205 (2011).

[25] F. Virot, R. Hayn, M. Richter, and J. van den Brink, Phys. Rev. Lett. 106, 236806 (2011).

[26] A. Delin, Phys. Rev. B 65, 153205 (2002).

[27] C.-X. Liu, X.-L. Qi, H. Zhang, X. Dai, Z. Fang, and S.-C. Zhang, Phys. Rev. B 82, 045122 (2010).

[28] R. Winkler, Spin-Orbit Coupling Effects in TwoDimensional Electron and Hole Systems (Springer, Berlin, 2003).

[29] E. G. Novik, A. Pfeuffer-Jeschke, T. Jungwirth, V. Latussek, C. R. Becker, G. Landwehr, H. Buhmann, and L. W. Molenkamp, Phys. Rev. B 72, 035321 (2005).

[30] G. Dresselhaus, Phys. Rev. 100, 580 (1955).

[31] M. Cardona, N. E. Christensen, and G. Fasol, Phys. Rev. Lett. 56, 2831 (1986).

[32] V. E. Sacksteder, S. Kettemann, Q. Wu, X. Dai, and Z. Fang, Phys. Rev. B 85, 205303 (2012).

[33] W.-Y. Shan, H.-Z. Lu, and S.-Q. Shen, New Journal of Physics 12, 043048 (2010).

[34] L. Fu, Phys. Rev. Lett. 103, 266801 (2009). 


\section{Appendix A: Kanel model Hamiltonian}

As long as the intrinsic bulk-inversion asymmetry of the zinc-blende crystal structure is not taken into account, the eight-band Kane model Hamiltonian reads

$$
\mathcal{H}=\left(\begin{array}{ccc}
\mathcal{H}^{6,6} & \mathcal{H}^{6,8} & \mathcal{H}^{6,7} \\
\cdot & \mathcal{H}^{8,8} & \mathcal{H}^{8,7} \\
\cdot & \cdot & \mathcal{H}^{7,7}
\end{array}\right)
$$

with the expression of the Hamiltonian subblocks $\mathcal{H}^{\alpha, \beta}$ listed in Table I They are expressed in terms of the usual Pauli matrices $\sigma_{x, y, z}$, the $J=3 / 2$ angular momentum matrices $J_{x}=\sqrt{3} / 2 \mathcal{I} \otimes \sigma_{x}+\left(\sigma_{x} \otimes \sigma_{x}+\sigma_{y} \otimes \sigma_{y}\right) / 2, J_{y}=$ $\sqrt{3} / 2 \mathcal{I} \otimes \sigma_{y}+\left(\sigma_{y} \otimes \sigma_{x}-\sigma_{x} \otimes \sigma_{y}\right) / 2, J_{z}=\sigma_{z} \otimes \mathcal{I}+\mathcal{I} \otimes \sigma_{z} / 2$ with $\mathcal{I}$ the identity matrix and the following $T_{i}$ matrices:

$$
\begin{aligned}
& T_{x}=\frac{1}{3 \sqrt{2}}\left(\begin{array}{cccc}
-\sqrt{3} & 0 & 1 & 0 \\
0 & -1 & 0 & \sqrt{3}
\end{array}\right) \\
& T_{y}=\frac{-i}{3 \sqrt{2}}\left(\begin{array}{cccc}
\sqrt{3} & 0 & 1 & 0 \\
0 & 1 & 0 & \sqrt{3}
\end{array}\right) \\
& T_{z}=\frac{\sqrt{2}}{3}\left(\begin{array}{llll}
0 & 1 & 0 & 0 \\
0 & 0 & 1 & 0
\end{array}\right) \\
& T_{x x}=\frac{1}{3 \sqrt{2}}\left(\begin{array}{cccc}
0 & -1 & 0 & \sqrt{3} \\
-\sqrt{3} & 0 & 1 & 0
\end{array}\right) \\
& T_{y y}=\frac{1}{3 \sqrt{2}}\left(\begin{array}{cccc}
0 & -1 & 0 & -\sqrt{3} \\
\sqrt{3} & 0 & 1 & 0
\end{array}\right) \\
& T_{z z}=\frac{\sqrt{2}}{3}\left(\begin{array}{cccc}
0 & 1 & 0 & 0 \\
0 & 0 & -1 & 0
\end{array}\right) \\
& T_{y z}=\frac{i}{2 \sqrt{6}}\left(\begin{array}{cccc}
-1 & 0 & -\sqrt{3} & 0 \\
0 & \sqrt{3} & 0 & 1
\end{array}\right) \\
& T_{z x}=\frac{1}{2 \sqrt{6}}\left(\begin{array}{cccc}
-1 & 0 & \sqrt{3} & 0 \\
0 & \sqrt{3} & 0 & -1
\end{array}\right) \\
& T_{x y}=\frac{i}{\sqrt{6}}\left(\begin{array}{cccc}
0 & 0 & 0 & -1 \\
-1 & 0 & 0 & 0
\end{array}\right)
\end{aligned}
$$

The parameters $F, \gamma_{1}, \gamma_{2}, \gamma_{3}$ describe the coupling to remote bands and are, as well as $P, E_{0}$, material-specific parameters. We have considered for simplicity the axial approximation $\bar{\gamma}=\left(\gamma_{2}+\gamma_{3}\right) / 2$ with the warping parameter $\mu=\left(\gamma_{3}-\gamma_{2}\right) / 2 \equiv 0$ in order to make the bulk band structure isotropic in the $k_{x, y}$ plane [29.

Effects of strain can be taken into consideration by applying the formalism of Bir and Pikus. They lead to additional terms in the eight-band Kane model Hamiltonian proportional to the strain tensor $\boldsymbol{\epsilon}$ and expressed in terms of the valence band deformation potentials $C_{1}, D_{u}, D_{u}^{\prime}$ [see TableII] The strain-induced interactions then lead to a change in the band-edges with the degeneracy at the $\Gamma$

\begin{tabular}{|c|c|c|c|}
\hline Hamilto & blocks & \multicolumn{2}{|c|}{$\mathbf{k} \cdot \mathbf{p}$ interactions } \\
\hline & & \multicolumn{2}{|c|}{$E_{0}+B(2 F+1) \mathbf{k}^{2}$} \\
\hline & & \multicolumn{2}{|c|}{$\sqrt{3} P \mathbf{T} \cdot \mathbf{k}$} \\
\hline & & \multicolumn{2}{|c|}{$-\frac{1}{\sqrt{3}} P \boldsymbol{\sigma} \cdot \mathbf{k}$} \\
\hline & & \multicolumn{2}{|c|}{$\begin{array}{c}-B \gamma_{1} \mathbf{k}^{2}+2 B \bar{\gamma}\left[\left(J_{x}^{2}-\frac{J^{2}}{3}\right) k_{x}^{2}+c . p .\right] \\
+B \bar{\gamma}\left[\left\{J_{x}, J_{y}\right\}\left\{k_{x}, k_{y}\right\}+c . p .\right]\end{array}$} \\
\hline & & \multicolumn{2}{|c|}{$\begin{array}{r}6 B \bar{\gamma}\left[\left(T_{x x}^{\dagger} k_{x}^{2}+\text { c.p. }\right)+\right. \\
\left.\left(T_{x y}^{\dagger}\left\{k_{x}, k_{y}\right\}+\text { c.p. }\right)\right]\end{array}$} \\
\hline & & \multicolumn{2}{|c|}{$-\Delta_{0}-B \gamma_{1} \mathbf{k}^{2}$} \\
\hline$E_{0}$ & $F$ & $P^{2} / B$ & $\gamma_{2}$ \\
\hline$-0.3 \mathrm{eV}$ & 0 & $18.8 \mathrm{eV}$ & 0.5 \\
\hline
\end{tabular}
point among the $\mathrm{LH}$ and $\mathrm{HH}$ bands that is preserved for a strain field with $\epsilon_{x x}+\epsilon_{y y}=2 \epsilon_{z z}$. This condition can be achieved, for instance by a simultaneous application
TABLE I: Expressions of the Kane model in the axial approximation. Here $\{A, B\}$ denotes the anticommutator for the $A, B$ operators, c.p. cyclic permutations of the preceding term and we defined $B=\hbar^{2} /\left(2 m_{0}\right)$ with $m_{0}$ the free electron mass. We also list the band structure parameters for $\mathrm{HgTe}$ of Ref. 29].

TABLE II: Strain-induced terms in the eight-band Kane model Hamiltonian

\begin{tabular}{cc}
\hline \hline Hamiltonian blocks & strain-induced interactions \\
\hline $\mathcal{H}^{6,6}$ & $C_{1} T r \epsilon$ \\
$\mathcal{H}^{8,8}$ & $D_{d} T r \epsilon+\frac{2}{3} D_{u}\left[\left(J_{x}^{2}-\frac{1}{3} J^{2}\right) \epsilon_{x x}+\right.$ c.p. $]$ \\
$\mathcal{H}^{7,7}$ & $\frac{2}{3} D_{u}^{\prime}\left[\left\{J_{x}, J_{y}\right\} \epsilon_{x y}+\right.$ c.p. $]$ \\
& $D_{d} T r \epsilon$ \\
\hline \hline
\end{tabular}

of two uniaxial stresses along the [100] ([010]) and [001] direction of magnitudes $X(Y)$ and $Z$ respectively with $X, Y=2 Z$ in which case $\operatorname{Tr} \epsilon=3\left(S_{11}+2 S_{12}\right) Z$ where $S_{11}, S_{12}$ are the elastic compliance constants.

\section{Appendix B: Surface state solutions}

We solve the eight-band Kane model Hamiltonian on the half-infinite space $z>0$ with open boundary conditions. We first consider our model Hamiltonian $\mathrm{Eq}, 3$ at the centre of the surface Brillouin zone $\left(k_{x, y} \equiv 0\right)$ where the degeneracy of the surface bands is protected by time-reversal symmetry. We therefore replace $k_{z} \rightarrow-i \partial_{z}$ and obtain the Schrödinger equation $\mathcal{H}_{k_{x, y} \equiv 0}\left(k_{z} \rightarrow-i \partial_{z}\right) \Psi(z) \equiv E_{\Gamma} \Psi(z)$ In the absence of BIS, the eigenstates take the form

$$
\Psi^{\uparrow}(z)=\left(\begin{array}{c}
\psi_{0}^{\uparrow} \\
\mathbf{0}
\end{array}\right) \quad \Psi^{\downarrow}(z)=\left(\begin{array}{c}
\mathbf{0} \\
\psi_{0}^{\downarrow}
\end{array}\right) .
$$


where $\psi_{0}^{\uparrow, \downarrow}$ is a three-dimensional spinor and $\mathbf{0}$ is a five component zero vector. Obviously to obtain the surface states, the wavefunction $\psi_{0}^{\uparrow, \downarrow}(z)$ should be localised at the [001] surface. We therefore put a trial spinorial wavefunction of the form $\psi_{0}^{\uparrow, \downarrow}(z)=\psi_{\lambda}^{\uparrow, \downarrow} \mathrm{e}^{\lambda z}$ into the Schrödinger equation thereby obtaining two eigenvalue equations with a unique secular equation the solution of which yields the general wavefunction solution

$$
\psi_{0}^{\uparrow, \downarrow}(z)=\sum_{\alpha=1}^{3} \sum_{\beta= \pm} C_{\alpha \beta} \psi_{\alpha \beta}^{\uparrow, \downarrow} \mathrm{e}^{\beta \lambda_{\alpha}\left(E_{\Gamma}\right) z} .
$$

The normalizability of the wavefunction in the $z>0$ region requires that the wavefunction contains only the three terms with $\beta$ negative and immediately yields the existence condition of the surface states $\mathcal{R}\left(\lambda_{\alpha}\right) \neq 0$ preventing the surface states to penetrate into the bulk and defines the decay length of the surface states $l_{c}=$ $\max \left\{1 / \mathcal{R}\left(\lambda_{\alpha}\right)\right\}$. Furthermore, applying the boundary condition $\psi_{0}^{\uparrow, \downarrow}(z=0) \equiv 0$, we obtain a secular equation [33] of the non-trivial solution for the coefficients $C_{\alpha \beta}$ that determines the energy of the two degenerate surface states at the surface Brillouin zone centre.

When the intrinsic BIS of the zinc-blende crystal structure is taken into account, the eigenstates of the Hamiltonian $\mathcal{H}_{k_{x, y} \equiv 0}$ have the form $\Psi^{\uparrow}(z)=\left(\psi_{0}^{\uparrow}, \mathbf{0}\right)^{T}$ and $\Psi^{\downarrow}(z)=\left(\mathbf{0}, \psi_{0}^{\downarrow}\right)^{T}$ with $\psi_{0}^{\uparrow, \downarrow}$ now a four-dimensional spinor. With this, the general solution can be written as $\psi_{0}^{\uparrow, \downarrow}(z)=\sum_{\alpha=1}^{4} \sum_{\beta= \pm} C_{\alpha \beta} \psi_{\alpha \beta}^{\uparrow, \downarrow} \mathrm{e}^{\beta \lambda_{\alpha}\left(E_{\Gamma}\right) z}$ with the condition of renormalizability of the wavefunction implying $\mathcal{R}\left(\lambda_{\alpha}\right) \neq 0$ and $\beta$ negative. For positive spin-splitting energy $\Delta_{0}$ the condition for the existence of the surface states is never verified since one of the $\lambda_{\alpha}$ is purely imaginary independent of the $c$ value. For negative spin-orbit splittings $\Delta_{0}$ instead, a renormalizable surface state solution can exist provided a non-trivial solution for the coefficients $C_{\alpha \beta}$ can be found.

\section{Appendix C: Two-dimensional k.p theory}

The point group of cubic mercury chalcogenides, is the group $T_{d}$, which does not contain the inversion operation. If one of these materials is terminated in the (001) direction, leading to a two-dimensional (001)-surface, the point group of that surface is reduced to $\mathcal{C}_{2 v}$, consisting of a twofold rotation symmetry along the $z$ axis, and two mirror symmetries $M_{x}: x \rightarrow-x$ and $M_{y}: y \rightarrow-y$ along the two diagonal $\Gamma-M$ directions of the surface BZ. By choosing as a natural basis for the Kramer doublet at the centre of the surface Brillouin zone, the total angular momentum $J= \pm 1 / 2$, we have that the twofold rotational symmetry can be represented as $C_{2}=-i \sigma_{z}$. Similarly we can represent the two mirror operations as $M_{x}=-i \sigma_{x}$ and $M_{y}=-i \sigma_{y}$ by choosing the phases of $\left|\psi_{\uparrow, \downarrow}\right\rangle$ appropriately. Finally the anti unitary time-reversal operator is represented as usual as $\mathcal{T}=i \sigma_{y} \mathcal{K}$. The Kramers doublet is split away from the centre of the surface BZ and the corresponding surface band structure can be studied within the k.p framework. The form of the effective surface Hamiltonian $\mathcal{H}(\mathbf{k})$ is highly constrained by timereversal and crystal symmetries. Indeed under $C_{2}$ and $M_{x, y}$, spin and momentum transform as follows:

$$
\begin{aligned}
C_{2} & : k_{x, y} \rightarrow-k_{x, y}, \sigma_{x, y} \rightarrow-\sigma_{x, y}, \sigma_{z} \rightarrow \sigma_{z} \\
M_{x} & : k_{x} \rightarrow-k_{x}, k_{y} \rightarrow k_{y}, \sigma_{x} \rightarrow \sigma_{x}, \sigma_{y, z} \rightarrow-\sigma_{y, z} \\
M_{y} & =M_{x}(x \rightarrow y)
\end{aligned}
$$

The Hamiltonian $\mathcal{H}(\mathbf{k})$ must be invariant under Eq 5 In addition, time-reversal symmetry gives the constraint

$$
\mathcal{H}(\mathbf{k})=\sigma_{y} \mathcal{H}^{\star}(-\mathbf{k}) \sigma_{y}
$$

As a result, we find that the effective Hamiltonian must take the following form up to second order in $\mathbf{k}$ :

$$
\mathcal{H}(\mathbf{k})_{2 v}=E_{2 v}(\mathbf{k}) \mathbb{I}+v_{F}^{x} k_{x} \sigma_{y}-v_{F}^{y} k_{y} \sigma_{x}
$$

where $E_{2 v}(\mathbf{k})=\hbar^{2} k_{x}^{2} /\left(2 m_{x}\right)+\hbar^{2} k_{y}^{2} /\left(2 m_{y}\right)$ generates particle-hole asymmetry. The Hamiltonian for the surface states at the (001) surface of a material with diamond crystal structure which instead possesses inversion symmetry can be easily calculated from Eq.7 by adding the additional constraint due to the four-fold rotational symmetry along the $z$ axis. As $\mathrm{C}_{4}$ can be represented as $\mathrm{e}^{i \pi \sigma_{z} / 4}$ we have that spin and momentum transform as

$$
C_{4}: k_{x} \rightarrow-k_{y}, k_{y} \rightarrow k_{x}, \sigma_{x} \rightarrow-\sigma_{y}, \sigma_{y} \rightarrow \sigma_{x} .
$$

As a result, the Hamiltonian for the corresponding bulk inversion symmetric material would read as

$$
\mathcal{H}(\mathbf{k})_{4 v}=E_{4 v}(\mathbf{k}) \mathbb{I}+v_{F}\left(k_{x} \sigma_{y}-k_{y} \sigma_{x}\right)
$$

where $E_{4 v}(\mathbf{k})=\hbar^{2} k^{2} /(2 m)$. Therefore systems with inversion symmetry display the same emerging $\mathcal{U}(1)$ rotational symmetry as is $\mathrm{Bi}_{2} \mathrm{Se}_{3}$ [34. 